\title{
Modeling and Analytical Simulation of High-Temperature Gas Filtration Combustion
}

\section{IBRAHIM, M; OLAYIWOLA, RO; COLE, AT; MD, SHEHU; MUHAMMAD, KM}

\author{
Department of Mathematics, \\ Federal University of Technology, Minna, Nigeria. \\ E-mail.: olaviwola.rasaq@futminna.edu.ng
}

\begin{abstract}
High temperature filtration in combustion and gasification processes is a highly interdisciplinary field. Thus, particle technology in general has to be supported by elements of physics, chemistry, thermodynamics and heat and mass transfer processes. Presented in this paper is the analytical method for describing hightemperature gas filtration combustion in an inert porous medium. We assume the porous media is highly permeable and both the contact time between the phases and the rate of oxidizer diffusion through the gas stream to the surface of the solid particles where the reaction occurs are not large. Also, we assume that the initial temperatures increase lengthwise. The coupled nonlinear partial differential equations describing the phenomenon have been decoupled using the parameter-expanding method and solved analytically using eigenfunctions expansion technique. The results obtained revealed that the combustion wave is propagated and oxidizer is consumed. A self-oscillating mode of gas filtration combustion was found with variation in the values of interfacial heat transfer. CJASEM

https://dx.doi.org/10.4314/jasem.v21i5.20
\end{abstract}

Keywords: Analytical method, filtration combustion, fuel, oxidizer, porous medium, temperature.

Filtration combustion, when an oxidizer is injected into a porous medium containing fuel, has numerous applications in technology and nature (enhanced oil recovery by in situ ombustion, coal gasification, selfpropagating high-temperature synthesis (SHS), smolder waves etc.). A High temperature, high pressure gas filtration is a fundamental component of several advanced coal-fired power systems ( Endo Kokubun et al., 2016).

Smoldering and SHS are both complicated processes involving chemistry; diffusive and convective transport of reactants, products, and heat through a porous medium; heat losses to the environment by radiation and convection (Wahle et al., 2003).

Mathematical models for filtration combustion of gases and condensed materials are based on the equations of the mechanics of multiphase media supplemented with the equations of chemical kinetics (Aldushin and Merzhanov, 1988). A fairly complete review of experimental and theoretical studies of filtration gas combustion in inert porous media is presented in (AbdulMujeebu et al., 2009). Numerical two-dimensional calculations of temperature and concentration fields supported experimentally have been performed in (Brenner et al., 2000) for combustion of a methane-air mixture in a rectangular burner with an inert coarse-pore section. Unsteady filtration combustion of gases in an inert porous layer is considered in (Prokof'ev et al., 2010) taking into account the gas pressure distribution in the pores. Due to the complexity of two-temperature models, very few results have been obtained. Wahle et al. (2003) employed a two-temperature model to study coflow FC in a sample open to gas flow only at the ends of the sample.
The objectives of this paper are to propose a twotemperature model for gas filtration combustion in an inert flat porous layer taking into account the gas pressure distribution in the pores, heat loss to the environment, energy accumulation, and net gas production in the reaction and to provide an approximated analytical solution to describe the phenomena.

\section{MATERIALS AND METHODS}

We consider a horizontal one-dimensional inert porous medium with an initially available concentration of a solid fuel. A combustion wave travels in the $\mathcal{X}$-direction through a sample of length $L$. The space variable is $x, 0<x<L$ and time $t$, $t>0$. Energy balances for each phase account for the heat flux through a given volume of the medium as well as heat exchange between the two phases. We assume the interphase heat transfer rate depends on the gas flux and follow Frank-Kamenetskii expression (Frank-Kamenetskii, 1969). The reaction rate depends on both the solid-fuel concentration and the oxidizer concentration in the gas at the surface of the solid particles where the reaction occurs. The kinetic function is assumed to be Arrhenius with large activation energy and the rate of oxidizer diffusion to the interface to be sufficiently large. We consider first-order solid-fuel reaction kinetic, so that $f(\eta)=1-\eta$. Based on the above assumptions, the two-temperature filtration gas combustion model consists of equations describing conservation of energy in each phase, gas mass, oxidizer mass, solid fuel mass and gas momentum as well as an equation of state and appropriate boundary and initial conditions as shown below: 
Conservation of energy of solid:

$(1-\phi) \rho_{s} c_{p s}\left(\frac{\partial T_{s}}{\partial t}+u \frac{\partial T_{s}}{\partial x}\right)=\frac{\partial}{\partial x}\left(\lambda_{s} \frac{\partial T_{s}}{\partial x}\right)-\alpha\left(T_{g}-T_{s}\right)-h\left(T_{s}-T_{0}\right)+(1-\phi) \rho_{f} Q k_{0} Y_{o x} e^{-\frac{E}{R T_{s}}}$

Conservation of energy of gas:

$$
\begin{gathered}
\phi \rho_{g} c_{p g}\left(\frac{\partial T_{g}}{\partial t}+u \frac{\partial T_{g}}{\partial x}\right)= \\
\mu_{g} c_{p g}\left(T_{g}-T_{s}\right)(1-\phi) \rho_{f} k_{0} Y_{o x} e^{-\frac{E}{R T_{s}}}
\end{gathered}
$$

Conservation of gas species (Oxygen):

$\frac{\partial}{\partial t}\left(\phi \rho_{g} Y_{o x}\right)+u \frac{\partial}{\partial x}\left(\phi \rho_{g} Y_{o x}\right)=-\frac{\partial}{\partial x}\left(\phi \rho_{g} v Y_{o x}\right)+\frac{\partial}{\partial x}\left(\phi \rho_{g} D \frac{\partial Y_{o x}}{\partial x}\right)-\mu_{0}(1-\phi) \rho_{f} k_{0} Y_{o x} e^{-\frac{E}{R T_{s}}}$

Solid fuel mass:

$\frac{\partial}{\partial t}\left((1-\phi) \rho_{f}\right)+u \frac{\partial}{\partial x}\left((1-\phi) \rho_{f}\right)=-(1-\phi) \rho_{f} k_{0} Y_{o x} e^{-\frac{E}{R T_{s}}}$

Gas mass:

$\frac{\partial}{\partial t}\left(\phi \rho_{g}\right)+u \frac{\partial}{\partial x}\left(\phi \rho_{g}\right)=-\frac{\partial}{\partial x}\left(\phi \rho_{g} v\right)+\mu_{g}(1-\phi) \rho_{f} k_{0} Y_{o x} e^{-\frac{E}{R T_{s}}}$

Darcy's Law:

$\frac{\partial P}{\partial x}+r v=0$

The equation of State:

$$
P=\rho_{g} \bar{R} T_{g}, \quad \bar{R}=\frac{R}{\phi M},
$$

where

$$
\alpha=\alpha_{0}\left(1+\left(\operatorname{Re} \cdot \frac{G}{G_{0}}\right)^{0.6} \operatorname{Pr}{ }^{0.3}\right)
$$

where $\phi$ is the porosity of the sample, $v$ is the gas velocity, $u$ is the propagation velocity, $E$ is activation energy, $R$ is the universal gas constant, $L$ is the length of sample, $\lambda$ is thermal conductivity, $Q$ is the enthalpy of reaction, $c$ is specific heat, $T$ is temperature, $Y$ is the mass fraction of the species, $t$ is time, $x$ is the spatial coordinate along the sample, $\alpha$ is the heat exchange rate between the two phases, $h$ is the coefficient of heat loss to the external environment, $D$ is the gas mass diffusivity, $r$ is the filtration resistance, $\mu_{g}$ is the net mass of gas produced $\left(\mu_{g}>0\right)$ or consumed $\left(\mu_{g}<0\right)$ per unit mass of fuel consumed in the reaction, $\mu_{0}$ is the mass stoichiometric coefficient for oxygen, $P$ is pressure, $\rho$ is density, $k_{0}$ is the pre-exponential factor, $M$ is the molecular weight of the gas, $G$ is the gas mass flux in the direction of propagation which can be expressed as $G=-\phi \rho_{g} v, \alpha_{0}$ is the interphase heat exchange rate when the gas is at rest with respect to the solid matrix, $\operatorname{Pr}$ is the Prandtl number, $\quad \operatorname{Re}_{0}$ is the Reynolds number corresponding to the gas flux at the inlet and the subscripts $s, g, f, p, o x, g p$ and 0 represent solid, gas, solidfuel, pressure, oxygen, gas product and initial respectively.

Coordinate Transformation

Here, we assume $\rho_{s}, \rho_{g}, \rho_{f}, c_{p}, c_{p g}, c_{p s}, \lambda_{s}, \lambda_{g}, \phi, D, \alpha$ to be constants and $\rho_{s}=\rho_{g}=\rho$.

By means of streamline function (Olayiwola, 2015),

$\eta(x, t)=\int_{0}^{x} \rho(s, t) d s$

The coordinate transformation becomes,

$\frac{\partial}{\partial x} \rightarrow \frac{\partial}{\partial \eta} \frac{\partial \eta}{\partial x}=\rho \frac{\partial}{\partial \eta}$
$\frac{\partial}{\partial t} \rightarrow \frac{\partial}{\partial \eta} \frac{\partial \eta}{\partial t}+\frac{\partial}{\partial t}=-\rho u \frac{\partial}{\partial \eta}+\frac{\partial}{\partial t}$

We make the additional assumptions that $\rho \lambda_{s}, \rho \lambda_{g}$ and $\rho^{2} D$ are constants. Although these assumptions could be relaxed in the future, they considerably simplify the equations.

Then, equations $(1)-(7)$ reduce to

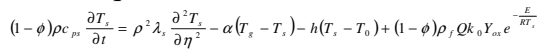

(11)

$$
\begin{gathered}
\phi \rho c_{p g} \frac{\partial T_{g}}{\partial t}=\phi \rho^{3} c_{p g} \frac{1}{r} \frac{\partial P}{\partial \eta} \frac{\partial T_{g}}{\partial \eta}+\rho^{2} \lambda_{g} \frac{\partial^{2} T_{g}}{\partial \eta^{2}}+\alpha\left(T_{g}-T_{s}\right)+ \\
\mu_{g} c_{p g}\left(T_{g}-T_{s}\right)(1-\phi) \rho_{f} k_{0} Y_{o x} e^{-\frac{E}{R T_{s}}} \\
\phi \rho \frac{\partial Y_{o x}}{\partial t}=\phi \rho^{3} \frac{\partial}{\partial \eta}\left(\frac{1}{r} \frac{\partial P}{\partial \eta} Y_{o x}\right)+\phi \rho^{3} D \frac{\partial^{2} Y_{o x}}{\partial \eta^{2}}-\mu_{0}(1-\phi) \rho_{f} k_{0} Y_{o x} e^{-\frac{E}{R T_{s}}}
\end{gathered}
$$

The initial and boundary conditions were formulated as follows:

Initial condition:

At $t=0$ and $\forall \eta$

$T_{s}=\frac{R T_{0}^{2}}{E}\left(1-\frac{\eta}{L}\right)+T_{0}, \quad T_{g}=\frac{R T_{0}^{2}}{E}\left(1-\frac{\eta}{L}\right)+T_{0}, \quad Y_{o x}=Y_{0}$

(14)

Boundary conditions: 


$$
\left.\begin{array}{ll}
\left.\frac{\partial T_{s}}{\partial \eta}\right|_{\eta=0}=0, & \left.\frac{\partial T_{s}}{\partial \eta}\right|_{\eta=L}=0 \\
\left.T_{g}\right|_{\eta=0}=T_{0}, & \left.\frac{\partial T_{g}}{\partial \eta}\right|_{\eta=L}=0 \\
\left.Y_{o x}\right|_{\eta=0}=Y_{0}, & \left.\frac{\partial Y_{o x}}{\partial \eta}\right|_{\eta=L}=0
\end{array}\right\},
$$

Method of Solution

In our analysis, we consider the pressure gradient to be parabolic (Olayiwola, 2012; Olayiwola et al., 2013), i.e.:

$\frac{\partial P}{\partial \eta}=f(\eta)=\frac{\eta}{L}\left(1-\frac{\eta}{L}\right)$

(16)

Non-dimensionalization

Here, we non-dimensionalized equations (11) - (16), using the following dimensionless variables:

$$
\left.\begin{array}{lr}
\eta^{\prime}=\frac{\eta}{L}, \quad t^{\prime}=\frac{t}{t_{0}}, & Y=\frac{Y_{o x}}{Y_{0}}, \quad \in=\frac{R T_{0}}{E} \\
\theta=\frac{E}{R T_{0}{ }^{2}}\left(T_{s}-T_{0}\right), & \varphi=\frac{E}{R T_{0}{ }^{2}}\left(T_{g}-T_{0}\right)
\end{array}\right\}
$$

where $t_{0}$ is the initial time for ignition to occur and we obtain and we obtain

$$
\frac{\partial \theta}{\partial t}=\lambda_{1} \frac{\partial^{2} \theta}{\partial \eta^{2}}-\alpha_{1}(\varphi-\theta)-h_{1} \theta+\delta Y \exp \left(\frac{\theta}{1+\in \theta}\right)
$$

$$
\frac{\partial \varphi}{\partial t}=\lambda_{2} \frac{\partial^{2} \varphi}{\partial \eta^{2}}+k \eta(1-\eta) \frac{\partial \varphi}{\partial \eta}+\alpha_{2}(\varphi-\theta)+\beta Y(\varphi-\theta) \exp \left(\frac{\theta}{1+\in \theta}\right)
$$

$$
\frac{\partial Y}{\partial t}=D_{1} \frac{\partial^{2} Y}{\partial \eta^{2}}+k \eta(1-\eta) \frac{\partial Y}{\partial \eta}+k(1-2 \eta) Y-\sigma Y \exp \left(\frac{\theta}{1+\in \theta}\right)
$$

(20)

Together with initial and boundary conditions:

$$
\left.\begin{array}{llll}
\theta(\eta, 0)=1-\eta, & \left.\frac{\partial \theta}{\partial \eta}\right|_{\eta=0}=0, & \left.\frac{\partial \theta}{\partial \eta}\right|_{\eta=1}=0 \\
\varphi(\eta, 0)=1-\eta, & \varphi(0, t)=0, & \left.\frac{\partial \varphi}{\partial \eta}\right|_{\eta=1}=0 \\
Y(\eta, 0)=1, & Y(0, t)=1, & \left.\frac{\partial Y}{\partial \eta}\right|_{\eta=1}=0
\end{array}\right\},
$$

(21)

where

$\lambda_{1}=\frac{\rho \lambda_{s} t_{0}}{L^{2}(1-\phi) c_{p s}}=$ Solid phase scaled thermal conductivity, $\lambda_{2}=\frac{\rho \lambda_{g} t_{0}}{L^{2} \phi c_{p g}}=$ Gas phase scaled thermal conductivity, $\alpha_{1}=\frac{\alpha t_{0}}{(1-\phi) \rho c_{p s}}, \quad \alpha_{2}=\frac{\alpha t_{0}}{\phi \rho c_{p g}}, \quad \delta=\frac{\rho_{f} k_{0} Q Y_{0} t_{0} e^{-\frac{E}{R T_{0}}}}{\rho c_{p s} \in T_{0}}=$ Frank-

Kamenetskii parameter, $h_{1}=\frac{h t_{0}}{(1-\phi) \rho c_{p s}}, k=\frac{\rho^{2} t_{0}}{r L}, \quad D_{1}=\frac{\rho^{2} t_{0} D}{L^{2}}=$ Species diffusion coefficient, $\quad \beta=\frac{\mu_{g} \rho_{f} k_{0}(1-\phi) t_{0} Y_{0} e^{-\frac{E}{R T_{0}}}}{\phi \rho}$, $\sigma=\frac{\mu_{0} \rho_{f} k_{0}(1-\phi) t_{0} e^{-\frac{E}{R T_{0}}}}{\phi \rho}$
Analytical Solution

We solve equations (18) - (21) using parameter-expanding method (where details can be found in (He, 2006)) and eigenfunctions expansion method (where details can be found in (Myint-U and Debnath, 1987)).

Ayeni (1982) has shown that $\exp \left(\frac{\theta}{1+\epsilon \theta}\right)$ can be approximated as $1+(e-2) \theta+\theta^{2} \quad$. In our analysis we are going to take an approximation of the form:

$\exp \left(\frac{\theta}{1+\in \theta}\right) \approx 1+(e-2) \theta$

so that equations (18) - (20) can be approximated as:

$\frac{\partial \theta}{\partial t}=\lambda_{1} \frac{\partial^{2} \theta}{\partial \eta^{2}}-\alpha_{1}(\varphi-\theta)-h_{1} \theta+\delta Y(1+(e-2) \theta)$

$\frac{\partial \varphi}{\partial t}=\lambda_{2} \frac{\partial^{2} \varphi}{\partial \eta^{2}}+k \eta(1-\eta) \frac{\partial \varphi}{\partial \eta}+\alpha_{2}(\varphi-\theta)+\beta Y(\varphi-\theta)(1+(e-2) \theta)$

(24)

$\frac{\partial Y}{\partial t}=D_{1} \frac{\partial^{2} Y}{\partial \eta^{2}}+k \eta(1-\eta) \frac{\partial Y}{\partial \eta}+k(1-2 \eta) Y-\sigma Y(1+(e-2) \theta)$

(25)

We let

$\alpha=r \in \quad k=p \in \quad \beta=q \in \quad \delta=a \in \quad \sigma=b \in$

Suppose that the solution of equations (23) - (25) can be expressed as:

$\theta(\eta, t)=\theta_{0}(\eta, t)+\in \theta_{1}(\eta, t)+\ldots$

$\varphi(\eta, t)=\varphi_{0}(\eta, t)+\in \varphi_{1}(\eta, t)+\ldots$

$Y(\eta, t)=Y_{0}(\eta, t)+\in Y_{1}(\eta, t)+\ldots$

Substituting (26) into (23) - (25) and processing, we obtain

$\frac{\partial \theta_{0}}{\partial t}=\lambda_{1} \frac{\partial^{2} \theta_{0}}{\partial \eta^{2}}-h_{1} \theta_{0}$

$\theta_{0}(\eta, 0)=1-\eta, \quad \theta_{0 \eta}(0, t)=0, \quad \theta_{0 \eta}(1, t)=0$

$\frac{\partial \varphi_{0}}{\partial t}=\lambda_{2} \frac{\partial^{2} \varphi_{0}}{\partial \eta^{2}}+\alpha_{2}\left(\varphi_{0}-\theta_{0}\right)$
$\varphi_{0}(\eta, 0)=1-\eta, \quad \varphi_{0}(0, t)=0, \quad \varphi_{0 \eta}(1, t)=0$

$\frac{\partial Y_{0}}{\partial t}=D_{1} \frac{\partial^{2} Y_{0}}{\partial \eta^{2}}$

$Y_{0}(\eta, 0)=0, \quad Y_{0}(0, t)=1, \quad Y_{0 \eta}(1, t)=0$

$\frac{\partial \theta_{1}}{\partial t}=\lambda_{1} \frac{\partial^{2} \theta_{1}}{\partial \eta^{2}}-r\left(\varphi_{0}-\theta_{0}\right)-h_{1} \theta_{1}+a Y_{0}\left(1+(e-2) \theta_{0}\right)$

(30)

$\theta_{1}(\eta, 0)=0, \quad \theta_{1 \eta}(0, t)=0, \quad \theta_{1 \eta}(1, t)=0$ 
$\frac{\partial \varphi_{1}}{\partial t}=\lambda_{2} \frac{\partial^{2} \varphi_{1}}{\partial \eta^{2}}+p \eta(1-\eta) \frac{\partial \varphi_{0}}{\partial \eta}+\alpha_{2}\left(\varphi_{1}-\theta_{1}\right)+q Y_{0}\left(\varphi_{0}-\theta_{0}\right)\left(1+(e-2) \theta_{0}\right)$

(31)

$$
\begin{aligned}
& \varphi_{1}(\eta, 0)=0, \quad \varphi_{1}(0, t)=0, \quad \varphi_{1 \eta}(1, t)=0 \\
& \frac{\partial Y_{1}}{\partial t}=D_{1} \frac{\partial^{2} Y_{1}}{\partial \eta^{2}}+p \eta(1-\eta) \frac{\partial Y_{0}}{\partial \eta}+p(1-2 \eta) Y_{0}-b Y_{0}\left(1+(e-2) \theta_{0}\right) \\
& Y_{1}(\eta, 0)=0, \quad Y_{1}(0, t)=0, \quad Y_{1 \eta}(1, t)=0
\end{aligned}
$$

Using eigenfunctions expansion method, we obtain the solution of equations (27) - (32) as

$$
\begin{aligned}
& \theta_{0}(\eta, t)=e^{-h_{1} t}+\sum_{n=1}^{\infty} A e^{-c t} \cos n \pi \eta \\
& \varphi_{0}(\eta, t)=\sum_{n=1}^{\infty}\left(B_{1}\left(e^{-h_{1} t}-e^{d t}\right)+\sum_{n=1}^{\infty} B_{2}\left(e^{-c t}-e^{d t}\right)+B e^{d t}\right) \sin \left(\frac{2 n-1}{2}\right) \pi \eta \\
& Y_{0}(\eta, t)=1+\sum_{n=1}^{\infty} A_{1} e^{-m t} \sin \left(\frac{2 n-1}{2}\right) \pi \eta \\
& \theta_{1}(\eta, t)=\theta_{10}(t)+\sum_{n=1}^{\infty} \theta_{1 n}(t) \cos n \pi \eta \\
& \varphi_{1}(\eta, t)=\sum_{n=1}^{\infty} s(t) \sin \left(\frac{2 n-1}{2}\right) \pi \eta \\
& Y_{1}(\eta, t)=\sum_{n=1}^{\infty} Q(t) \sin \left(\frac{2 n-1}{2}\right) \pi \eta
\end{aligned}
$$

where

$$
\begin{aligned}
& \theta_{10}(t)=\frac{2 a}{h_{1}}\left(1-e^{-h_{1} t}\right)+2 a(e-2) t e^{-h_{1} t}+\sum_{n=1}^{\infty} p_{1}\left(e^{-m t}-e^{-h_{1} t}\right)-(e-2) \sum_{n=1}^{\infty} p_{1}\left(e^{-\left(h_{1}+m\right) t}-e^{-h_{1} t}\right)- \\
& (e-2) \sum_{n=1}^{\infty} A_{1} \sum_{n=1}^{\infty} p_{2}\left(e^{-(c+m) t}-e^{-h_{1} t}\right)+2 r t e^{-h_{1} t}+ \\
& r \sum_{n=1}^{\infty} A_{1}\left(\begin{array}{l}
B_{1}\left(t e^{-h_{1} t}-p_{3}\left(e^{d t}-e^{-h_{1} t}\right)\right)+p_{4}\left(e^{d t}-e^{-h_{1} t}\right)- \\
\sum B_{2}\left(p_{5}\left(e^{-c t}-e^{-h_{1} t}\right)-p_{3}\left(e^{-d t}-e^{-h_{1} t}\right)\right)
\end{array}\right) \\
& \theta_{1 n}(t)=a(e-2) \sum_{n=1}^{\infty} A t e^{-c t}-\sum_{n=1}^{\infty} p_{6}\left(e^{-m t}-e^{-c t}\right)-(e-2) \sum_{n=1}^{\infty} p_{7}\left(e^{-\left(h_{1}+m\right) t}-e^{-c t}\right)- \\
& (e-2) \sum_{n=1}^{\infty} A_{1} \sum_{n=1}^{\infty} p_{8}\left(e^{-(c+m) t}-e^{-c t}\right)+r \sum_{n=1}^{\infty} A t e^{-c t}- \\
& r \sum_{n=1}^{\infty} A_{2}\left(\begin{array}{l}
B_{1}\left(p_{9}\left(e^{-h_{1} t}-e^{-c t}\right)-p_{10}\left(e^{d t}-e^{-c t}\right)\right)+p_{11}\left(e^{d t}-e^{-c t}\right)- \\
\sum_{n=1}^{\infty} B_{2}\left(t e^{-c t}-p_{10}\left(e^{-d t}-e^{-h_{1} t}\right)\right)
\end{array}\right) \\
& Q(t)=p \sum_{n=1}^{\infty} q_{1} t e^{-m t}+p\left(q_{2}\left(1-e^{-m t}\right)-\sum_{n=1}^{\infty} q_{3} t e^{-m t}\right)-b\left(A_{1}\left(1-e^{-m t}\right)+\sum_{n=1}^{\infty} A_{1} t e^{-m t}\right)- \\
& b(e-2)\left(q_{4}\left(e^{-h_{1} t}-e^{-m t}\right)+\sum_{n=1}^{\infty} q_{5}\left(e^{-c t}-e^{-m t}\right)-\sum_{n=1}^{\infty} q_{6}\left(e^{-\left(h_{1}+m\right) t}-e^{-m t}\right)\right) \\
& s(t)=e(t)+e_{1}(t)+e_{2}(t)-e_{3}(t)-e_{4}(t)-e_{5}(t)-e_{6}(t) \\
& e(t)=q \sum_{n=1}^{\infty}\left(B_{1}\left(p_{3}\left(e^{-h_{1} t}-e^{d t}\right)-t e^{d t}\right)+B t e^{d t}-\sum_{n=1}^{\infty} B_{2}\left(-p_{10}\left(e^{-h_{1} t}-e^{d t}\right)-t e^{d t}\right)\right) \\
& e_{1}(t)=q \sum_{n=1}^{\infty} A_{1} A_{3} \sum_{n=1}^{\infty}\left(\begin{array}{l}
B_{1}\left(-q_{7}\left(e^{-\left(m+h_{1}\right) t}-e^{d t}\right)+\frac{1}{m}\left(e^{(d-m) t}-e^{d t}\right)\right)-\frac{B}{m}\left(e^{(d-m) t}-e^{d t}\right)- \\
\sum_{n=1}^{\infty} B_{2}\left(-q_{8}\left(e^{-(m+c) t}-e^{d t}\right)+\frac{1}{m}\left(e^{(d-m) t}-e^{d t}\right)\right)
\end{array}\right) \\
& e_{2}(t)=q(e-2)\left(r_{1}(t)+r_{2}(t)+r_{3}(t)\right) \\
& r_{1}(t)=\sum_{n=1}^{\infty}\left(\begin{array}{l}
B_{1}\left(-q_{9}\left(e^{-\left(2 h_{1}\right) t}-e^{d t}\right)+\frac{1}{h_{1}}\left(e^{\left(d-h_{1}\right) t}-e^{d t}\right)\right)-\frac{B}{h_{1}}\left(e^{\left(d-h_{1}\right) t}-e^{d t}\right)- \\
\sum_{n=1}^{\infty} B_{2}\left(-q_{10}\left(e^{-\left(h_{1}+c\right) t}-e^{d t}\right)+\frac{1}{h_{1}}\left(e^{\left(d-h_{1}\right) t}-e^{d t}\right)\right)
\end{array}\right)
\end{aligned}
$$

\section{RESULTS AND \\ DISCUSSION}

We solve the systems of coupled nonlinear partial differential equations describing hightemperature gas filtration combustion in an inert porous medium analytically. We decouple the equations using parameterexpanding method and solve the resulting equations using eigenfunctions expansion technique. Using (33) - (38) in (26), analytical solutions of equations (18) - (21) are computed for the values of $\delta=04, \lambda_{1}=01, \lambda_{2}=01, \quad D=01, \in=01$ $k=1, \sigma=02, \quad \beta=01, \quad h_{1}=4, \alpha_{1}=0.00025 \alpha_{2}=0.125$ . The following figs. explain the temperatures and species mass fraction distribution against different dimensionless parameters.

Fig. 1 shows the effect of FrankKamenetskii number $(\delta)$ on the solid phase temperature. It is observed that the solid phase temperature decreases as time increases and decrease along distance but increase with increase in Frank-Kamenetskii number.

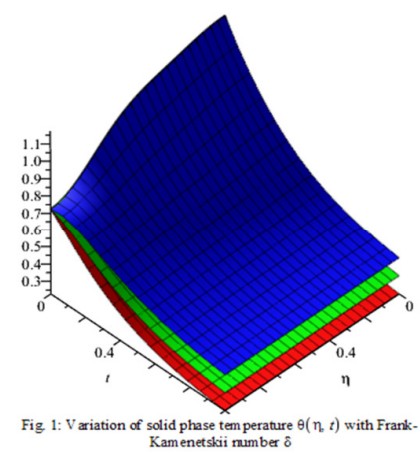

Fig. 2 depicts the effect of oxygen mass diffusivity $\left(D_{1}\right)$ on the oxidizer mass. It is observed that the oxidizer mass increases as time increases and behave sinusoidally along distance but decrease with increase in oxygen mass diffusivity. 


$$
\begin{array}{r}
r_{2}(t)=\sum_{n=1}^{\infty} A_{1} A_{3} \sum_{n=1}^{\infty}\left(\begin{array}{c}
B_{1}\left(-q_{11}\left(e^{-\left(m+2 h_{1}\right) t}-e^{d t}\right)+\frac{1}{m+h_{1}}\left(e^{\left(d-m-h_{1}\right) t}-e^{d t}\right)\right)- \\
\frac{B}{m+h_{1}}\left(e^{\left(d-m-h_{1}\right) t}-e^{d t}\right)-\sum_{n=1}^{\infty} B_{2}\left(\begin{array}{c}
-q_{12}\left(e^{-\left(m+h_{1}+c\right) t}-e^{d t}\right)+ \\
\frac{1}{m+h_{1}}\left(e^{\left(d-m-h_{1}\right) t}-e^{d t}\right)
\end{array}\right)
\end{array}\right) \\
r_{3}(t)=\sum_{n=1}^{\infty} A_{1} \sum_{n=1}^{\infty} A \sum_{n=1}^{\infty} B_{4}\left(\begin{array}{c}
B_{1}\left(-q_{12}\left(e^{-\left(m+c+h_{1}\right) t}-e^{d t}\right)+\frac{1}{m+c}\left(e^{(d-m-c) t}-e^{d t}\right)\right)- \\
\frac{B}{m+c}\left(e^{(d-m-c) t}-e^{d t}\right)-\sum_{n=1}^{\infty} B_{2}\left(\begin{array}{c}
-q_{13}\left(e^{-(m+2 c) t}-e^{d t}\right)+ \\
\frac{1}{m+c}\left(e^{(d-m-c) t}-e^{d t}\right)
\end{array}\right)
\end{array}\right)
\end{array}
$$$$
e_{3}(t)=q\left(-q_{14}\left(e^{-h_{1} t}-e^{d t}\right)+\sum_{n=1}^{\infty} q_{15}\left(e^{-c t}-e^{d t}\right)-\sum_{n=1}^{\infty} q_{16}\left(e^{-\left(m+h_{1}\right) t}-e^{d t}\right)\right)
$$$$
e_{4}(t)=q(e-2)\left(\begin{array}{l}
-q_{17}\left(e^{-2 h_{1} t}-e^{d t}\right)+2 \sum_{n=1}^{\infty} q_{18}\left(e^{-\left(c+h_{1}\right) t}-e^{d t}\right)+\sum_{n=1}^{\infty} A \sum_{n=1}^{\infty} q_{19}\left(e^{-2 c t}-e^{d t}\right)- \\
\sum_{n=1}^{\infty} q_{20}\left(e^{-\left(m+2 h_{1}\right) t}-e^{d t}\right)--\frac{1}{2} \sum_{n=1}^{\infty} A_{1} \sum_{n=1}^{\infty} A \sum_{n=1}^{\infty} q_{21}\left(e^{-(m+2 c) t}-e^{d t}\right)
\end{array}\right)
$$$$
e_{5}(t)=p \sum_{n=1}^{\infty} A_{4}\left(B_{1}\left(-q_{22}\left(e^{-h_{1} t}-e^{d t}\right)-t e^{d t}\right)+B t e^{d t}-\sum_{n=1}^{\infty} B_{2}\left(-q_{23}\left(e^{-c t}-e^{d t}\right)-t e^{d t}\right)\right)
$$$$
e_{6}(t)=\alpha_{2}\left(A_{1}\left(r_{4}(t)+r_{5}(t)+r_{6}(t)-r_{7}(t)-r_{8}(t)+r_{9}(t)+r_{10}(t)-r_{11}(t)\right)\right)
$$$$
r_{4}(t)=\frac{2 a}{h_{1}}\left(-\frac{1}{d}\left(1-e^{d t}\right)+\frac{1}{d+h_{1}}\left(e^{-h_{1} t}-e^{d t}\right)\right)
$$$$
r_{5}(t)=2 a(e-2)\left(\frac{1}{\left(d+h_{1}\right)^{2}}\left(e^{-h_{1} t}-e^{d t}\right)-\frac{1}{d+h_{1}} t e^{-h_{1} t}\right)
$$$$
r_{6}(t)=\sum_{n=1}^{\infty} q_{24}\left(\frac{1}{(d+m)}\left(e^{-m t}-e^{d t}\right)-\frac{1}{d+h_{1}}\left(e^{-h_{1} t}-e^{d t}\right)\right)
$$$$
r_{7}(t)=(e-2) \sum_{n=1}^{\infty} q_{25}\left(-\frac{1}{\left(d+m+h_{1}\right)}\left(e^{-\left(h_{1}+m\right) t}-e^{d t}\right)+\frac{1}{d+h_{1}}\left(e^{-h_{1} t}-e^{d t}\right)\right)
$$$$
r_{8}(t)=(e-2) \sum_{n=1}^{\infty} A_{1} \sum_{n=1}^{\infty} q_{26}\left(-\frac{1}{(d+m+c)}\left(e^{-(c+m) t}-e^{d t}\right)+\frac{1}{d+h_{1}}\left(e^{-h_{1} t}-e^{d t}\right)\right)
$$$$
r_{9}(t)=2 r\left(\frac{1}{\left(d+h_{1}\right)^{2}}\left(e^{-h_{1} t}-e^{d t}\right)-\frac{1}{d+h_{1}} t e^{-h_{1} t}\right)
$$$$
\left(B_{1}\left(\begin{array}{l}
\left(\frac{1}{\left(d+h_{1}\right)^{2}}\left(e^{-h_{1} t}-e^{d t}\right)-\frac{1}{d+h_{1}} t e^{-h_{1} t}\right) \\
\frac{1}{d+h_{1}}\left(t e^{d t}+\frac{1}{\left(d+h_{1}\right)}\left(e^{-h_{1} t}-e^{d t}\right)\right)
\end{array}\right)+\right.
$$$$
r_{10}(t)=r \sum_{n=1}^{\infty} A_{1} \frac{B}{d+h_{1}}\left(t e^{d t}+\frac{1}{\left(d+h_{1}\right)}\left(e^{-h_{1} t}-e^{d t}\right)\right)-
$$$$
\sum_{n=1}^{\infty} B_{2}\left(\begin{array}{l}
\frac{1}{h_{1}-c}\left(-\frac{1}{d+c}\left(e^{-c t}-e^{d t}\right)+\frac{1}{d+h_{1}}\left(e^{-h_{1} t}-e^{d t}\right)\right)- \\
\frac{1}{d+h_{1}}\left(t e^{d t}+\frac{1}{\left(d+h_{1}\right)}\left(e^{-h_{1} t}-e^{d t}\right)\right)
\end{array}\right)
$$$$
r_{11}(t)=\sum_{n=1}^{\infty} A_{2}\left(r_{12}(t)-r_{13}(t)-r_{14}(t)-r_{15}(t)+r_{16}(t)-r_{17}(t)\right)
$$$$
r_{12}(t)=a(e-2) \sum_{n=1}^{\infty} A\left(\frac{1}{(d+c)^{2}}\left(e^{-c t}-e^{d t}\right)-\frac{1}{d+c} t e^{-c t}\right)
$$$$
r_{13}(t)=\sum_{n=1}^{\infty} q_{27}\left(-\frac{1}{(d+m)}\left(e^{-m t}-e^{d t}\right)+\frac{1}{d+c}\left(e^{-c t}-e^{d t}\right)\right)
$$

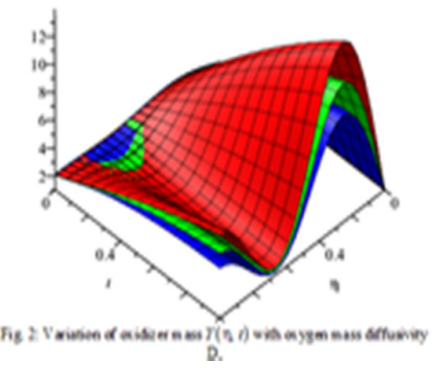

Fig. 3 presents the effect of filtration resistance $(k)$ on the oxidizer mass. It is observed that the oxidizer mass increases as time increases and behave sinusoidally along distance but increase with increase in filtration resistance.

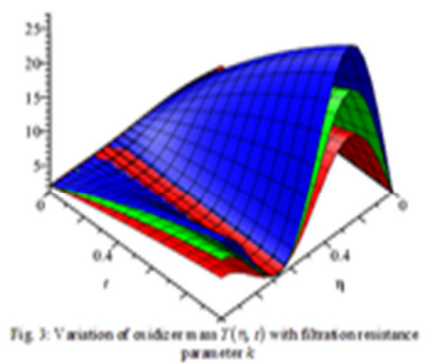

Fig. 4 displays the effect of heat loss parameter $\left(h_{1}\right)$ on the solid phase temperature. It is observed that the solid phase temperature decreases as time increases and decrease along distance but decrease with increase in heat loss to the environment.

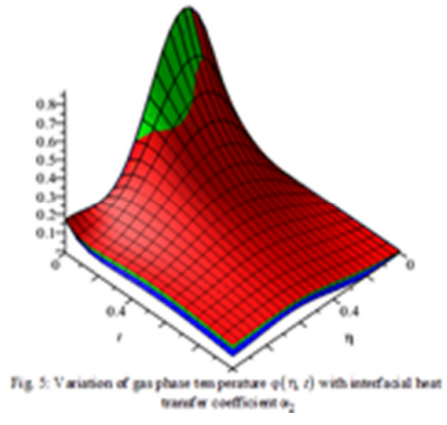

Fig. 5 shows the effect of interfacial heat transfer coefficient $\left(\alpha_{2}\right)$ on the gas phase temperature. It is observed that the gas phase temperature decreases as time increases and decrease along 


$$
\begin{aligned}
& r_{14}(t)=(e-2) \sum_{n=1}^{\infty} q_{28}\left(-\frac{1}{\left(d+m+h_{1}\right)}\left(e^{-\left(m+h_{1}\right) t}-e^{d t}\right)+\frac{1}{d+c}\left(e^{-c t}-e^{d t}\right)\right) \\
& r_{15}(t)=(e-2) \sum_{n=1}^{\infty} A_{1} \sum_{n=1}^{\infty} q_{29}\left(-\frac{1}{(d+m+c)}\left(e^{-(m+c) t}-e^{d t}\right)+\frac{1}{d+c}\left(e^{-c t}-e^{d t}\right)\right) \\
& r_{16}(t)=r \sum_{n=1}^{\infty} A\left(-\frac{1}{(d+c)^{2}}\left(e^{-c t}-e^{d t}\right)-\frac{1}{d+c} t e^{-c t}\right) \\
& B_{1}\left(\begin{array}{l}
\frac{1}{c-h_{1}}\left(\frac{1}{\left(d+h_{1}\right)}\left(e^{-h_{1} t}-e^{d t}\right)+\frac{1}{(d+c)}\left(e^{-c t}-e^{d t}\right)\right)- \\
\frac{1}{(d+c)}\left(t e^{d t}+\frac{1}{(d+c)}\left(e^{-c t}-e^{d t}\right)\right)
\end{array}\right)+ \\
& r_{17}(t)=r \sum_{n=1}^{\infty} A_{2} \frac{B}{(d+c)}\left(t e^{d t}+\frac{1}{(d+c)}\left(e^{-c t}-e^{d t}\right)\right)- \\
& \sum_{n=1}^{\infty} B_{2}\left(\begin{array}{l}
\left(\frac{1}{(d+c)^{2}}\left(e^{-c t}-e^{d t}\right)-\frac{1}{(d+c)} t e^{-c t}\right)- \\
\frac{1}{(d+c)}\left(t e^{d t}+\frac{1}{(d+c)}\left(e^{-c t}-e^{d t}\right)\right)
\end{array}\right) \\
& A=-\frac{2(-1)^{n}}{n^{2} \pi^{2}}, \quad c=h_{1}+\lambda_{1} n^{2} \pi^{2}, \quad d=\alpha_{2}-\lambda_{2} n^{2} \pi^{2}, \quad B=\frac{4\left(2 n \pi-\pi+2(-1)^{n}\right)}{(2 n-1)^{2} \pi^{2}}, \\
& A_{1}=\frac{4}{(2 n-1) \pi}, \quad m=\frac{1}{4} D_{1}(2 n-1)^{2} \pi^{2}, \quad A_{2}=\frac{4(2 n-1)}{(4 n-1) \pi}, \quad B_{3}=\frac{4\left(1-4 n-4 n^{2}\right)}{24 n^{3}-4 n^{2}-6 n+1}, \\
& A_{3}=\frac{8}{3(2 n-1) \pi}, \quad B_{4}=\frac{4\left(48 n^{3}-72 n^{2}+36 n-6\right)}{\left(128 n^{3}-176 n^{2}+72 n-9\right) \pi}, \quad B_{1}=\frac{4 \alpha_{2}}{(2 n-1) \pi\left(d+h_{1}\right)}, \\
& B_{2}=\frac{4 A \alpha_{2}(2 n-1)}{(4 n-1) \pi(d+c)}, \quad A_{4}=\frac{2(2 n-1)}{\left(8 n^{3}-12 n^{2}+6 n-1\right) \pi^{2}} \\
& p_{1}=\frac{A_{1}^{2}}{\left(h_{1}-m\right)}, \quad p_{2}=\frac{A A_{2}}{\left(h_{1}-m-c\right)}, \quad p_{3}=\frac{1}{\left(h_{1}+d\right)}, \quad p_{4}=\frac{B}{\left(h_{1}+d\right)}, \quad p_{5}=\frac{1}{\left(h_{1}-c\right)}, \\
& p_{6}=\frac{A_{1} A_{2}}{(c-m)}, \quad p_{7}=\frac{A_{1} A_{2}}{\left(c-h_{1}-m\right)}, \quad p_{8}=\frac{A B_{3}}{m}, \quad p_{9}=\frac{1}{\left(c-h_{1}\right)}, \quad p_{10}=\frac{1}{(c+d)}, \quad p_{11}=\frac{B}{(c+d)} \\
& q_{1}=\frac{2 \pi A_{1}}{(2 n-1)^{2}}, \quad q_{2}=\frac{4\left((2 n-1) \pi+4(-1)^{n}\right)}{(2 n-1)^{2} \pi^{2} m}, \quad q_{3}=\frac{4 A_{1}}{(2 n-1)^{2} \pi^{2}}, \quad q_{4}=\frac{A_{1}}{\left(m-h_{1}\right)}, \\
& q_{5}=\frac{A A_{2}}{(m-c)}, \quad q_{6}=\frac{A_{1}}{h_{1}}, \quad q_{7}=\frac{1}{\left(d+m+h_{1}\right)}, \quad q_{8}=\frac{1}{(d+m+c)}, \quad q_{9}=\frac{1}{\left(d+2 h_{1}\right)}, \\
& q_{10}=\frac{1}{\left(c+d+h_{1}\right)}, \quad q_{11}=\frac{1}{\left(m+d+2 h_{1}\right)}, \quad q_{12}=\frac{1}{\left(m+d+c+h_{1}\right)}, \quad q_{13}=\frac{1}{(m+d+2 c)} \\
& q_{14}=\frac{A_{1}}{\left(d+h_{1}\right)}, \quad q_{15}=\frac{A A_{2}}{(d+c)}, \quad q_{16}=\frac{A_{1}}{\left(d+m+h_{1}\right)}, \quad q_{17}=\frac{A_{1}}{\left(d+2 h_{1}\right)}, \quad q_{18}=\frac{A A_{2}}{\left(d+h_{1}+c\right)}, \\
& q_{19}=\frac{A B_{3}}{d+2 c}, \quad q_{20}=\frac{A_{1}}{\left(d+m+2 h_{1}\right)}, \quad q_{21}=\frac{A}{(d+m+2 c)}, \quad q_{22}=\frac{1}{\left(d+h_{1}\right)}, \quad q_{23}=\frac{1}{(c+d)}, \\
& q_{24}=\frac{A_{1}^{2}}{\left(h_{1}-m\right)}, \quad q_{25}=\frac{A_{1}^{2}}{m}, \quad q_{26}=\frac{A A_{2}}{\left(h_{1}-m-c\right)}, \quad q_{27}=\frac{A_{1} A_{2}}{(c-m)}, \quad q_{28}=\frac{A_{1} A_{2}}{\left(c-m-h_{1}\right)}, \quad q_{29}=\frac{A B_{3}}{m}
\end{aligned}
$$

The computations were done using computer symbolic algebraic package MAPLE

The results of an analytical investigation of the effect of FrankKamenetskii number, oxygen mass diffusivity, filtration resistance, heat loss to the environment and interfacial heat transfer coefficient physically means that the combustion wave is propagated and oxidizer is consumed. A self-oscillating mode of gas filtration combustion was found with variation in the values of interfacial heat transfer parameter.

Conclusion: We have formulated and solved analytically a mathematical model of filtration combustion of gases in an inert porous medium with an initially available concentration of a solid fuel to determine the concentration and temperature distributions. We decoupled the equations usingparameter expanding method and solved the resulting equations using Eigen functions expansion technique. Finally, we have provided the graphical summaries of the system responses.

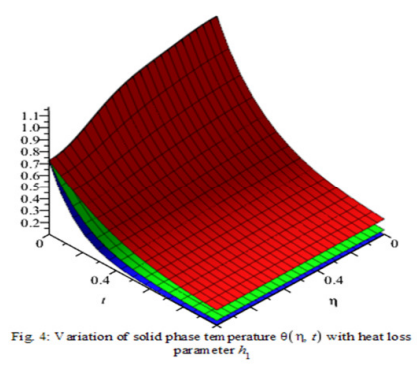

\section{REFERENCES}

AbdulMujeebu, M; Abdullah, MZ; AbuBakar, MZ; A. A. Mohamad; R. M. N. Muhad; M. K. Abdullah (2009). Combustion in porous media and its applications - A comprehensive survey. $J$. Environ. Manag. 90: 22872312.

Aldushin, AP; Merzhanov, AG (1988). Theory of filtration combustion: general concepts and status of research. in Propagation of Heat Waves in Heterogeneous Media [in Russian], Nauka, Novosibirsk. 9-52.

Ayeni, RO (1982). On the explosion of Chain-thermal Reactions. J. Austral. Math. Soc. (Series B). 24: 194-202.

Brenner, G; Pickenacker, K; Pickenacker, O (2000). Numerical and experimental investigation of atrixstabilized methane air combustion in porous inert media. Combust. Flame, 123: 201-213.

Endo Kokubun, MA; Khoshnevis Gargar, N; Bruinning, H; Mailybaev, AA (2016). Multicomponent effects in liquid-gas filtration combustion. Combustion and Flame. 169: 51-62

Frank-Kamenetskii, DA (1969). Diffusion and Heat Transfer in Chemical Kinetics. Plenum Press, New York.

He, JH (2006). Some asymptotic methods for strongly nonlinear 
equations. Int. J. Modern Phys. B. 20(10): 1141 - 1199.

Myint-U, T; Debnath, L (1987). Partial Differential Equation for Scientists and Engineers. PTR Prentice - Hall, Englewood Cliffs, New Jersey 07632.

Olayiwola, RO (2012). Effects of Some Thermophysical Properties on the Propagation of forward and Opposed Smouldering Combustion. The Pacific Journal of Science and Technology. 13(2): 108 - 118. http://www.akamaiuniversity.us/PJST.htm

Olayiwola, RO; Olatunji, SO; Ajao, SO; Waheed, AA; Lanlege, DI (2013). Effect of Frank-Kamenetskii Parameter on the Propagation of forward and Opposed Smouldering Combustion. Journal of the Nigerian Association of Mathematical Physics, 23: 203 - 214.
Prokof'ev, VG; Kirdyashkin, AI; Salamatov, VG; Smolyakov, VK (2010). Unsteady Combustion of Gases in an Inert Porous Layer. Combustion, Explosion and Shock Waves. 46: 641 - 646.

Olayiwola, RO (2015). Modeling and Simulation of Combustion Fronts in Porous Media. Journal of Nigeria Mathematical Society. 2 (1): $100-103$.

Wahle, C; Matkowsky, B; Aldushin, F (2003). An Effects of Gas-Solid Nonequilibrium in Filtration Combustion. Combust. Sci. Tech. 175: 1389-1499. 